\title{
Normalized transfer function for characterizing conversion efficiency and harmonic distortion of all-optical sampling
}

\author{
ZHANG ShangJian*, LIN ChangYu, LI Xuan, ZOU ZhiTai, LIU Yong \& LIU YongZhi \\ State Key Laboratory of Electronic, Thin Film and Integrated Devices, School of Optoelectronic Information, University of Electronic Science \& \\ Technology of China, Chengdu 610054, China
}

Received June 9, 2010; accepted October 9, 2010; published online April 13, 2011

\begin{abstract}
A novel method for characterizing the nonlinearity of all-optical sampling is proposed based on the normalized transfer function. Simulation results demonstrate the effectiveness of our method. Furthermore, an all-optical sampling experiment is performed to verify our method. Both simulation and experiment show consistency between our method and the measurements. The method only requires normalization and polynomial fitting of the transfer curve, and enables direct expression of the nonlinearity with the coefficients of the normalized transfer function.
\end{abstract}

optical signal processing, all-optical sampling, linearity, nonlinear distortion

Citation: Zhang S J, Lin C Y, Li X, et al. Normalized transfer function for characterizing conversion efficiency and harmonic distortion of all-optical sampling. Chinese Sci Bull, 2011, 56: 1633-1636, doi: 10.1007/s11434-010-4272-3

Nonlinearity of all-optical sampling is very critical to the resolution of all-optical analog-to-digital conversion [1-3], and distortion analysis is needed to achieve a good sampling performance. In most cases, conversion efficiency and harmonic distortion are introduced to characterize the nonlinearity and optimize the sampling performance [4,5]. The conversion efficiency represents the signal promotion, while the harmonic distortion reflects the signal distortion. In traditional spectrum measurement, a sinusoidal signal is input as the analog signal to be sampled, and the harmonics of the output signal are measured for comparison, from which the nonlinearity can be evaluated [6-8]. This method is very accurate; however, the measurement should be repeated for both input and output signals once the input signal is updated, which is not suitable for properly choosing the operating parameters in advance.

Prior to this work, we reported the concept of a nonlinear transfer function, with which the harmonics of the output signal at any input sine or cosine signal can be calculated through function composition [8]. The method avoids repeatable spec-

*Corresponding author (email: sjzhang@uestc.edu.cn) trum measurement; however, the Fourier expansion of the function composition leads to non-uniform expression and redundant computation. In this paper, we propose a normalized transfer function which can avoid both spectrum measurement and function composition. The consistency between our method and the function composition method is investigated in the simulation. Furthermore, the method is applied to characterize the all-optical sampling experiment, and is compared with spectrum measurement to check the accuracy.

\section{Theoretical description}

As is shown in Figure 1, the transfer function can be obtained by a polynomial fitting of the transfer curve and written as

$$
y_{i}=h\left(x_{i}\right)=\sum_{k=0}^{m} a_{k} x_{i}^{k}, i=1, \cdots, n,
$$

where $x_{i}$ and $y_{i}\left(i=1,2, \cdots, n\right.$ and $\left.x_{1}<x_{2}<\cdots<x_{n}\right)$ represent the intensity of the input analog signal and that of the output 


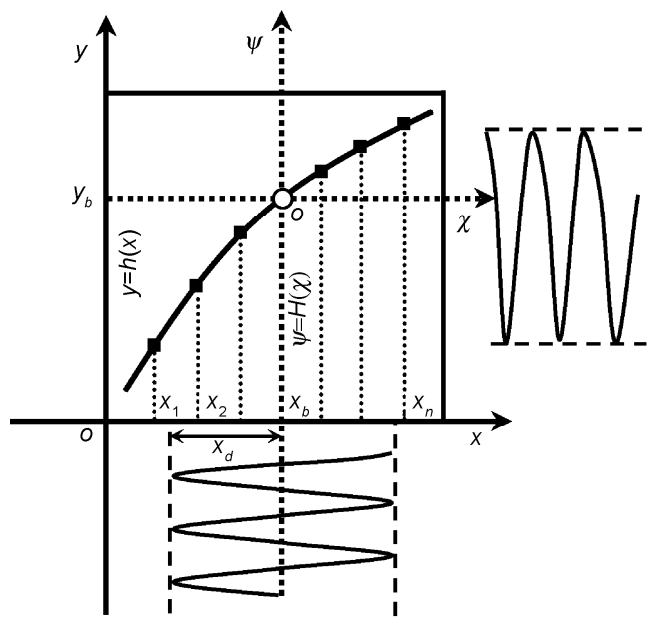

Figure 1 Schematic of the normalized transfer function.

probe signal. In the case of $m=1$, the sampling is linear, whereas in the case of $m>1$, nonlinearity will occur and give rise to distortion in all-optical sampling.

We assume the input analog signal to be sampled is a cosine signal as follows,

$$
x(t)=x_{b}+x_{d} \cos \omega t,
$$

where $x_{b}$ is the bias power and $x_{d}$ is the modulation amplitude. Substituting eq. (2) into eq. (1), the output signal can be obtained as

$$
y(t)=h(x(t))=\sum_{k=0}^{m} a_{k}\left(x_{b}+x_{d} \cos \omega t\right)^{k} .
$$

To simplify the Fourier expansion of the function composition, we propose a normalized transfer function: the origin of the $x$ oy coordinate is translated to $\left(x_{b}, y_{b}\right)$, and both the $x$-axis and $y$-axis are normalized with the same ratio of $1 / x_{d}$. In the new coordinate $\chi \circ \psi$, the input signal is $\chi(t)=$ $\cos \omega t$, and the output probe signal can be simplified to

$$
\psi(t)=H(\chi(t))=\sum_{k=0}^{m} \alpha_{k} \cos ^{k} \omega t,
$$

where $\alpha_{k}(k=0,1, \cdots, m)$ are the coefficients of the normalized transfer function. To observe the harmonics, we need to further translate the power of the trigonometric function into a multiple-angle function with the power-reduction formulas as follows $[9,10]$,

$$
\begin{aligned}
& \cos ^{2 n} \theta=\frac{1}{2^{2 n-1}}\left[\sum_{k=0}^{n-1} C_{2 n}^{k} \cos (2 n-2 k) \theta+\frac{1}{2} C_{2 n}^{n}\right], \\
& \cos ^{2 n+1} \theta=\frac{1}{2^{2 n}}\left[\sum_{k=0}^{n} C_{2 n+1}^{k} \cos (2 n-2 k+1) \theta\right] .
\end{aligned}
$$

Thus, we have

$$
\psi(t)=H(\cos \omega t)=\sum_{k=0}^{m} \rho_{k} \cos k \omega t,
$$

$$
\rho_{k}=\sum_{i=0}^{[(m-k) / 2]} \frac{\alpha_{k+2 i} C_{k+2 i}^{i}}{2^{k+2 i-1}},
$$

where $[(m-k) / 2]$ gives the greatest integer less than or equal to $(m-k) / 2$. Therefore, the conversion efficiency and harmonic distortion can be obtained as

$$
e_{f}=\rho_{1}, d_{k}=\left|\rho_{k} / \rho_{1}\right|, k=2,3, \cdots, m .
$$

The method basically includes three steps: (i) Translate $\left(x_{i}, y_{i}\right)$ of xoy into $\left(\chi_{i}, \psi_{i}\right)$ of $\chi o \psi$ through $\chi_{i}=\left(x_{i}-x_{b}\right) / x_{d}$ and $\psi_{i}=\left(y_{i}-y_{b}\right) / x_{d}$; (ii) Construct the transfer function $\psi=\sum \alpha_{k} \chi^{k}$ from the normalized transfer curve $\psi_{i}=H\left(\chi_{i}\right)$; and (iii) Calculate the conversion efficiency $e_{f}$ and harmonic distortion $d_{k}$ with the coefficients $\alpha_{k}$. From eqs. (7) and (8), the novelty of our method lies in the fact that the nonlinearity only depends on the polynomial coefficients and the Fourier expansion becomes avoidable.

\section{Experiment and results}

As shown in Figure 2, we first test our method with a simulated transfer curve that is cited from [8]. In the normalized coordinate, the polynomial transfer function is

$$
\psi=H(\chi)=\sum_{k=0}^{4} \alpha_{k} \chi^{k},
$$

where the polynomial coefficients are $\alpha_{0}=0.0118, \alpha_{1}=$ $2.3647, \alpha_{2}=-1.6519, \alpha_{3}=0.5229, \alpha_{4}=-0.0441$. With the polynomial coefficients, we have

$$
\begin{gathered}
\rho_{1}=\alpha_{1} C_{1}^{0}+\alpha_{3} C_{3}^{1} / 4=2.7569, \\
\rho_{2}=\alpha_{2} C_{2}^{0} / 2+\alpha_{4} C_{4}^{1} / 8=-0.8480, \\
\rho_{3}=\alpha_{3} C_{3}^{0} / 4=0.1307, \\
\rho_{4}=\alpha_{4} C_{4}^{0} / 8=0.0055 .
\end{gathered}
$$

Substituting the coefficients $\rho_{k}$ into eq. (8), the conversion efficiency and harmonic distortion can be obtained and are

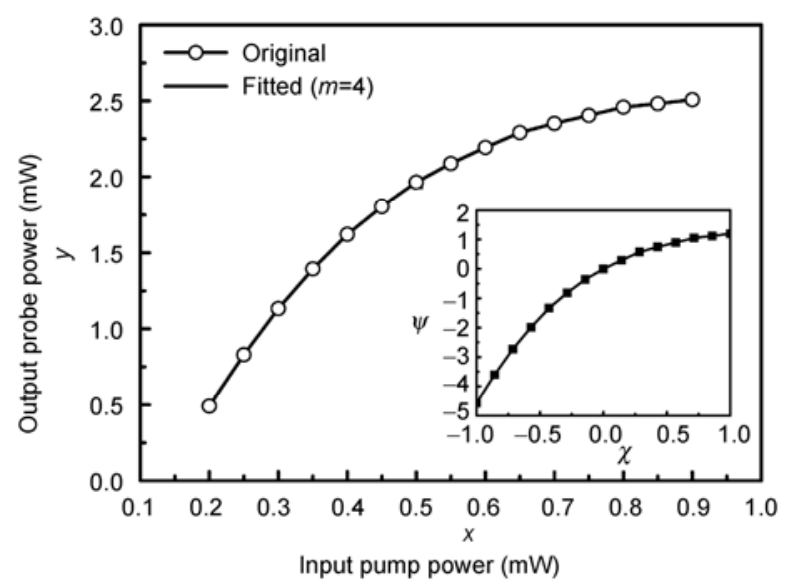

Figure 2 Normalization for the transfer curve of all-optical sampling. 
Table 1 Simulated results from our method (I) and [8](II)

\begin{tabular}{ccccc}
\hline & $e_{f}$ & $d_{2}$ & $d_{3}$ & $d_{4}$ \\
\hline I & 2.7569 & 0.3076 & 0.0474 & 0.0024 \\
II & 2.7571 & 0.3082 & 0.0482 & 0.0026 \\
\hline
\end{tabular}

listed in Table 1, where the results in [8] are also shown for comparison. The agreement of the results indicates the consistency of both methods. Moreover, our method omits Fourier expansion and leads to a more concise calculation.

As shown in Figure 3, in our experiment a mode-locked fiber ring laser $\left(\lambda_{b}=1550.20 \mathrm{~nm}\right)$ emits $1.4 \mathrm{ps}$ pulses at 40 $\mathrm{GHz}$. The pulse train with an average power of $-1.3 \mathrm{dBm}$ is injected into the semiconductor optical amplifier (SOA) as the probe signal. An optical cosine signal at $2.5 \mathrm{GHz}$ is coupled into the SOA as the pump signal. The SOA is operated at a bias current of $276 \mathrm{~mA}$. The all-optical sampling scheme can be found in $[11,12]$, here we only focus on the experimental results.

The measured and normalized transfer curves are shown in Figure 4(a) and (b). The polynomial coefficients of the normalized transfer function are $\alpha_{0}=-0.0024, \alpha_{1}=3.1156$, $\alpha_{2}=-0.4888, \alpha_{3}=-0.4457, \alpha_{4}=0.1961$, with which the conversion efficiency and the harmonic distortion can be directly obtained and are listed in Table 2 . To check the accuracy of our method, both the waveform and the spectrum of the output probe signal are also measured and shown in Figure 5. The nonlinearity from the spectrum measurement is also listed in Table 2 for comparison. The difference, which is less than $5 \%$, is mainly because the truncation error of the limited order of the polynomial function. The difference could be further decreased by increasing the order of the polynomial function, but it does not affect the optimization of the operating parameters of all-optical sampling.

\section{Conclusions}

We have proposed a normalized transfer function to characterize the nonlinear distortion of all-optical sampling. Different from the traditional method and our previous

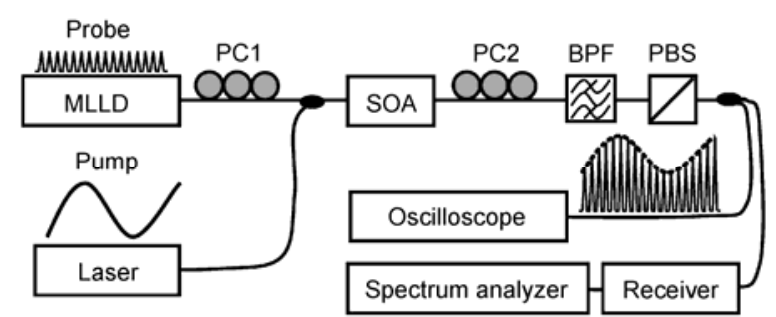

Figure 3 Schematic of all-optical sampling based on nonlinear polarization rotation. SOA, Semiconductor optical amplifier; MLLD, mode lock laser diode; PC, polarization controller; BPF, optical band pass filter; PBS, polarization beam splitter.
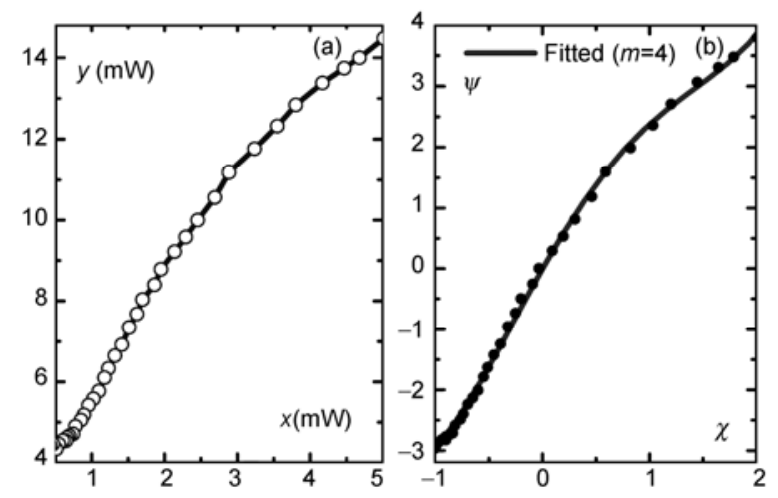

Figure 4 (a) Measured transfer curve and (b) its normalized transfer function.

Table 2 Experimental results with our method (I) and spectrum measurement (II)

\begin{tabular}{ccccc}
\hline & $e_{f}$ & $d_{2}$ & $d_{3}$ & $d_{4}$ \\
\hline I & 2.7813 & 0.0526 & 0.0386 & 0.0088 \\
II & 2.6425 & 0.0547 & 0.0372 & 0.0086 \\
\hline
\end{tabular}

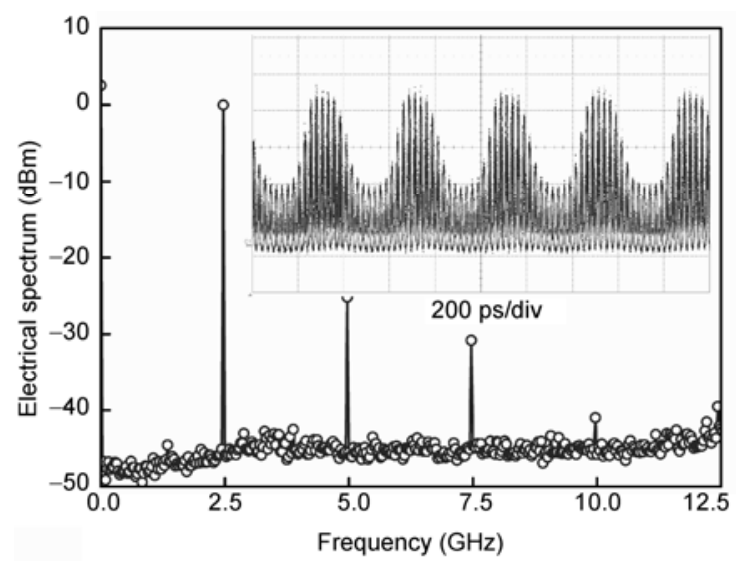

Figure 5 Measured spectrum of the output probe signal. Inset: Waveform of the output probe signal.

work, our method enables a uniform expression of conversion efficiency and harmonic distortion in terms of the polynomial coefficients, which avoids both spectrum measurement and Fourier expansion. Both simulated and experimental results show consistency between our method and measurement. The method is simple and useful for distortion characterization and parameters optimization of alloptical sampling.

This work was supported by the National Natural Science Foundation of China (60736038, 60907008 and 60925019), and the Education Ministry Funds for New Teachers (200806141102 and 20090185120027).

1 Valley G C. Photonic analog-to-digital converters. Opt Express, 2007, 15: 1955-1982

2 Juodawlkis P W, Twichell J C, Betts G E, et al. Optically sampled 
analog-to-digital converters. IEEE Trans Microwave Theory Tech, 2001, 49: 1840-1853

3 Wang M H, Qi W, Yu H, et al. Research of high speed optical switch based on compound semiconductor. Chinese Sci Bull, 2009, 54: 3679-3684

4 Shim J, Liu B, Piprek J, et al. Nonlinear properties of traveling-wave electroabsorption modulator. IEEE Photon Technol Lett, 2004, 16: 1035-1037

5 Yang J Z, Yu C H, Liu C W. A new method for power signal harmonic analysis. IEEE Trans Power Deliv, 2005, 20: 1235-1239

6 Chung Y D, Kang Y S, Choi K S, et al. Analog characteristics of electroabsorption modulator for RF/optic conversion; RF gain and IMD3. Microwave Opt Technol Lett, 2005, 48: 1151-1155

7 Liu Y, Chen S F, Wang X, et al. Overall optimization of high-speed semiconductor laser modules. Chinese Sci Bull, 2009, 54: 3697-3703
8 Zhang S J, Liu Y, Zhang Q S, et al. Characterization on linearity and conversion efficiency of all-optical sampling based on semiconductor optical amplifier (in Chinese). Acta Opt Sin, 2009, 29: 2529-2533

9 Writing Group of Mathematical Handbook. Mathematical Handbook(in Chinese). Beijing: Higher Education Press, 2004

10 Abramowitz M, Stegun I A. Handbook of Mathematical Functions with Formulas, Graphs, and Mathematical Tables. New York: Dover Publications, 1972

11 Zhang S J, Liu Y, Zhang Q S, et al. All-optical sampling based on nonlinear polarization rotation in semiconductor optical amplifiers. $\mathrm{J}$ Optoelectron Biomed Mater, 2009, 1: 339-344

12 Duan P X, Chen L G, Zhang S J, et al. All-optical 2R regeneration based on self-induced polarization rotation in a single semiconductor optical amplifier. Chinese Sci Bull, 2009, 54: 3704-3708

Open Access This article is distributed under the terms of the Creative Commons Attribution License which permits any use, distribution, and reproduction in any medium, provided the original author(s) and source are credited. 